\title{
The Spinning Jenny and the Industrial Revolution: A Reappraisal
}

\author{
Ugo GragnOlati, DANIELE MOSCHELla, AND \\ EMANUELE PUGLIESE
}

W hy was the Industrial Revolution British? In a recent article published in this JOURNAL, Robert Allen argues that only in England was the price of labor relative to capital high enough to justify the adoption of the labor-saving technologies which characterized the Industrial Revolution. ${ }^{1}$ To support his argument, he uses the spinning jenny as a case study. The jenny was indeed an important labor-saving technology that was invented and widely adopted in England but not in France. Allen explains this fact by calculating the returns to adopting the jenny in each country: according to his calculations the jenny was profitable in England but not in France.

The present note shows that Allen's conclusions rest on implausible profitability computations. In particular, Allen assumes that output remains constant after the adoption of the jenny while hours worked decrease dramatically. From a theoretical perspective, this is equivalent to an assumption that hours worked move inversely with the marginal product of labor. As soon as these restrictive assumptions are abandoned, the jenny turns out being profitable both in England and in France. Hence the mystery of the adoption of the jenny during the Industrial Revolution remains.

\section{PROFITABILITY COMPUTATIONS}

The most profitable between two alternative techniques of production is the one generating a higher net present value. Accordingly, a general formula for the profitability of the jenny (indexed by $J$ ) relative to the spinning wheel (indexed by $S$ ) would read ${ }^{2}$

$$
K_{J}=\sum_{t=1}^{T} \frac{p\left(q_{J}-q_{S}\right)-w\left(L_{J}-L_{S}\right)-m_{J}}{(1+r)^{t}}
$$

On the side of outflows, $K$ is the upfront cost of capital and $m$ its yearly maintenance cost, while $w$ is the daily wage paid for each of the $L$ work days in a year. Inflows are instead constituted by the price $p$ obtained for each of the $q$ units of output sold in a year. Finally, $T$ is the life time of the jenny and the unknown $r$ is the rate of return

The Journal of Economic History, Vol. 71, No. 2 (June 2011). (C) The Economic History Association. All rights reserved. ISSN 0022-0507.

Ugo Gragnolati, Daniele Moschella, and Emanuele Pugliese are Ph.D. Candidates, LEM, Scuola Superiore Sant'Anna, Piazza Martiri della Libertà 33, 56127, Pisa, Italy. E-mails: u.gragnolati@sssup.it,d.moschella@sssup.it, and e.pugliese@sssup.it.

The authors wish to thank Alessandro Nuvolari for his support, suggestions, and patience. All authors share equal responsibilities for the present work.

${ }^{1}$ Allen, "Spinning Jenny." See also Allen, Global Perspective and "Scientific Revolution."

${ }^{2}$ Following Allen, we keep things simple by neglecting the purchase price and the maintenance costs of the spinning wheel. 
from choosing the jenny over the spinning wheel. Hence, spinners will adopt the jenny if the rate of return $r$ that solved equation 1 is higher than the prevailing rate of interest.

Starting from equation 1, two alternative assumptions can be made to carry out viable profitability computations given the available data. Before moving to them, the following notation is introduced. Each technology is characterized by a labor input coefficient $\alpha$ such that $\alpha \cdot q=L$; consequently, $\alpha^{-1}$ is the labor productivity of the technology in question and the term $P=\alpha_{S} / \alpha_{J}$ is the labor productivity of the jenny relative to the spinning wheel.

\section{Scenario 1: Fixed Output and Decreased Labor}

To reach the specific profitability computation used by Allen, output has to be assumed constant. In fact, when $q_{S}=q_{J}$ the revenues from the two production techniques are the same and no longer figure in the profitability calculation that only depends on differences in labor input. The amount of labor supplied by the spinner has to decrease with the adoption of the jenny by an amount equal to the gain in relative efficiency generated by the jenny its self. ${ }^{3}$ If the spinner uses $L_{S}$ units of labor with a spinning wheel to produce $q=L_{S} / \alpha_{S}$, then she can produce the same $q$ with $\alpha_{J} q$ units of labor and a jenny. Hence, $L_{J}=L_{S^{*}} \alpha_{J} / \alpha_{S}$. Defining $L_{S}=Y \cdot D$, where $Y$ is the number of working days in a year and $0<D \leq 1$ is the part time fraction that workers devote to spinning cotton with the spinning wheel, implies that $L_{J}=Y \cdot D \cdot \alpha_{J} / \alpha_{S}$. It then follows that the profitability formula for fixed ouput is

$$
K_{J}=\sum_{t=1}^{T} \frac{w \cdot Y \cdot D\left(\frac{\alpha_{S}-\alpha_{J}}{\alpha_{S}}\right)-m_{J}}{(1+r)^{t}}
$$

Equation 2 is exactly the one used by Allen to assess the profitability of the spinning jenny across different countries, which are characterized by a different value of $K_{J}$ and $w$. Crucially, the value of $r$ as obtained with equation 2 is flawed in two distinct regards.

First, the assumption of fixed output is implausible when discussing the adoption of a new technology. In fact, a reduction of marginal costs would lead any profitmaximizing producer to increase output. Not surprisingly, this is what happened also to cotton spinners during the Industrial Revolution: as Allen himself recalls, "Producers were paid by [...] the pound that they spun, and they bought jennies to increase their production and thus their earnings."4

Second, equation 2 provides a downward biased estimate of profitability. Indeed, precisely because labor inputs fall, the utilization rate of capital drops after adoption. In effect, spinners are forced to buy more capital than they will ever use: this reduces the profitability of the jenny by inflating capital costs. An example clarifies the point. Suppose, like Allen does, that a cotton spinner worked 100 full working days in a year, and set this level of capital utilization to be 100 percent. ${ }^{5}$ If we maintained the fixed output assumption, what would the spinner do if the jenny turned out profitable

\footnotetext{
3 "Spinning Jenny," p. 915, eq. 2.

${ }^{4}$ Ibid., p. 915.

${ }^{5}$ Ibid., p. 916.
} 
with $P=3$ ? She would substitute her one spinning wheel with one jenny to end up producing the same fixed output in one third of the time. Hence, the adoption of the jenny would lead her to devote only 33.3 full working days per year to spinning! In parallel, the utilization rate of capital would drop to 33.3 percent, while for the remaining 66.6 percent of the time the jenny would be left idle. Hence, Allen's computation shows how profitable it is to buy a jenny that is kept it in the closet for 332 days a year while being used in the remaining 33 days. It would seem likely that even if the original spinner only works 33 days, she would want to either hire another spinner to work on the machine, or rent out the machine to someone else.

\section{Scenario 2: Fixed Labor and Increased Output}

It is possible to carry out a profitability computation that does not suffer from the limitations discussed above. Let us turn for a moment to some considerations on the price of output. An analysis of the profitability of the jenny relative to the spinning wheel is relevant only if $p \geq w \alpha_{S}$. Otherwise, if $p<w \alpha_{S}$, spinning wheels would be out of business since the variable cost $w \alpha_{S}$ would exceed the marginal revenue $p$. Then, if price must be such that $p \geq w \alpha_{S}$, it follows that $p>w \alpha_{S}$ would guarantee higher revenues as compared to $p=w \alpha_{S}$ for any technique in use. Under these considerations, setting $p=w \alpha_{S}$ is a "safe" assumption since it cannot overestimate the profitability of the jenny. Hence, equation 1 becomes

$$
K_{J}=\sum_{t=1}^{T} \frac{w \cdot\left(\frac{\alpha_{S}-\alpha_{J}}{\alpha_{J}}\right) L_{J}-m_{J}}{(1+r)^{t}}
$$

Notably, according to equation 3 , the value of $r$ is increasing in $L_{J}$. Therefore, it would not be surprising if cotton spinners during the Industrial Revolution decided to substitute labor for leisure, as argued by Jan De Vries: working more would make the adoption of the new technology more profitable. ${ }^{6}$ Nonetheless, labor supply will be here assumed to remain constant after adoption in order to make results especially robust and transparent, hence $L_{J}=L_{S}=L=Y D$. Under this assumption, equation 3 becomes

$$
K_{J}=\sum_{t=1}^{T} \frac{w \cdot\left(\frac{\alpha_{S}-\alpha_{J}}{\alpha_{J}}\right) Y \cdot D-m_{J}}{(1+r)^{t}}
$$

Notably, equation 4 differs from equation 2 by the term $P=\alpha_{S} / \alpha_{J}$, which is the labor productivity of the jenny relative to the spinning wheel. More precisely, multiplying $w$ in equation 2 by $P$ yields exactly equation 4 . It must also be noticed that equation 4 could be obtained even without any specific assumption on the value of $p$. In particular, equation 4 can be recovered expressing per unit of output both inflows and outflows of equation 1 . This is equivalent to imputing exactly as much capital services as those actually enjoyed by the adopter, contrarily to what happens with equation 2 .

\footnotetext{
${ }^{6}$ De Vries, "Industrious Revolution."
} 
TABLE 1

DATA FOR ENGLAND AND FRANCE

\begin{tabular}{lrr}
\hline \hline Variables & England & France \\
\hline$w$ (per day) & $6.25 d$. & 9 st. \\
$Y$ (days) & 250 & 250 \\
$K_{J}$ & $840 d$. & $2800 s t$. \\
$\mu$ & $1 / 10$ & $1 / 10$ \\
$m_{J}=K_{J} \cdot \mu$ & $84 d$. & 280 st. \\
$K_{S}$ & $12 d . \approx 0$ & 24 st. $\approx 0$ \\
$m_{S}=K_{S} \cdot \mu$ & $1.2 d . \approx 0$ & $2.4 s t . \approx 0$ \\
$T$ (years) & 10 & 10 \\
\hline
\end{tabular}

Notes: Money values are expressed in pence (d.) and sous tournois (st.). Variables: $w$, daily wage; $Y$, working days in a year; $K_{J}$, purchase price of the jenny; $\mu$, yearly maintenance rate; $m_{J}$, yearly maintenance cost of the jenny; $K_{S}$, purchase price of the spinning wheel; $m_{J}$, yearly maintenance cost of the spinning wheel; and $T$, years of life of the jenny.

Source: All data from Allen, "Spinning Jenny," p. 916.

\section{RESULTS}

The profitability of the jenny relative to the spinning wheel is computed here according to the two different scenarios discussed above. The necessary data are taken directly from Allen, as summarized by Table 1. Given these data, the values of $r$ as computed with equations 2 and 4 are reported in Table 2. The results are exposed for varying values of the part time fraction $D$ and of the productivity of the jenny relative to the spinning wheel $P$, identically to what Allen does in his work. Moreover, Table 2 shows explicitly the level of $L_{J}$ implied by the assumptions on labor supply specific to each scenario.

The values of $r$ obtained under SCENARIO 1 are systematically lower than those under SCENARIO 2. The third and fourth column of Table 2 report the downward biased values of $r$ obtained by Allen using equation 2, while the sixth and seventh column report those obtained with equation 4. Notice that the results in SCENARIO 1 are based on implausibly low levels of yearly working days (fifth column of Table 2), which imply unreasonably low utilization rates of the jenny. As soon as SCENARIO 1 is abandoned and SCENARIO 2 is considered, the jenny becomes always profitable both in England and France. In particular, under SCENARIO 2, $r$ exceeds the expected rate of return on alternative activities, which Allen deems to be 15 percent. $^{7}$ This is always true in both countries but in the totally unlikely case of $P=2$ and $D<0.4$. To realize how remote this case is, consider that other authors suggest much higher ranges of $P$ compared to the one investigated by Allen; for instance, David Landes estimates the productivity of the jenny relative to the spinning wheel to be "anywhere from six up to twenty-four to one for the jenny." ${ }^{8}$ Notice that also a third scenario would be economically reasonable: one in which spinners decided to work more with the jenny than they did with the spinning wheel precisely to increase the profitability of adopting the former. In that case, profitabilities would be even higher since $r$ is increasing in $L_{J}$.

\footnotetext{
${ }^{5}$ Allen, "Spinning Jenny," p. 917.

${ }^{6}$ Landes, Unbound Prometheus, p. 85.
} 
TABLE 2

THE PROFITABILITY OF THE JENNY IN ENGLAND AND FRANCE

\begin{tabular}{|c|c|c|c|c|c|c|c|}
\hline \multirow[b]{2}{*}{$P$} & \multirow[b]{2}{*}{$D$} & \multicolumn{3}{|c|}{$\begin{array}{c}\text { Scenario } 1 \\
\text { Fixed Output }\end{array}$} & \multicolumn{3}{|c|}{$\begin{array}{c}\text { Scenario } 2 \\
\text { Fixed Labor Input }\end{array}$} \\
\hline & & $r_{U K}$ & $r_{F R}$ & $L_{J}$ & $r_{U K}$ & $r_{F R}$ & $L_{J}$ \\
\hline 2 & 0.3 & 12.3 & -21.7 & 37.5 & 44.6 & 6.8 & 75.0 \\
\hline 2 & 0.4 & 24.0 & -8.2 & 50.0 & 63.9 & 17.9 & 100.0 \\
\hline 2 & 0.5 & 34.6 & 0.2 & 62.5 & 82.8 & 27.5 & 125.0 \\
\hline 3 & 0.3 & 24.0 & -8.2 & 25.0 & 101.5 & 36.5 & 75.0 \\
\hline 3 & 0.4 & 38.0 & 2.5 & 33.3 & 138.8 & 53.5 & 100.0 \\
\hline 3 & 0.5 & 51.2 & 10.7 & 41.7 & 176.0 & 70.0 & 125.0 \\
\hline 4 & 0.3 & 29.4 & -3.7 & 18.7 & 157.4 & 61.8 & 75.0 \\
\hline 4 & 0.4 & 44.7 & 6.8 & 25.0 & 213.2 & 86.2 & 100.0 \\
\hline 4 & 0.5 & 59.2 & 15.3 & 31.2 & 269.0 & 110.2 & 125.0 \\
\hline
\end{tabular}

\section{CONCLUSION}

Differentials in the price of labor relative to capital are insufficient to explain why the spinning jenny spread in England but not in France. The quantitative assessments carried out in the present work reveal that the jenny was profitably adoptable in both countries, despite their difference in terms of relative prices. Nonetheless, it was James Hargraeves and not some French inventor to develop the jenny. This implies that the potential demand for innovation might be necessary but nonsufficient to generate a corresponding supply of inventions. The riddle of the different fortunes of the spinning jenny in England and in France during the Industrial Revolution remains open.

\section{REFERENCES}

Allen, Robert C. "The Industrial Revolution in Miniature: The Spinning Jenny in Britain, France, and India." University of Oxford, Department of Economics, Economics Series Working Papers, No. 375, 2007.

. The British Industrial Revolution in Global Perspective. Cambridge: Cambridge University Press, 2009a.

. "The Industrial Revolution in Miniature: The Spinning Jenny in Britain,

France, and India." The Journal of Economic History 69, no.4 (2009b): 901-27.

"Why the Industrial Revolution was British: Commerce, Induced Innovation,

and the Scientific Revolution." Economic History Review, forthcoming. 
De Vries, Jan. "The Industrial Revolution and the Industrious Revolution." The Journal of Economic History 54, no. 2 (1994): 249-70.

Landes, David. The Unbound Prometheus: Technological Change and Industrial Development in Western Europe from 1750 to the Present. Cambridge: Cambridge University Press, 1969. 